\title{
Challenges and facilitators for early career re- searchers completing systematic or scoping re- views in the health sciences: A scoping review protocol
}

\section{Background}

An increasing number of systematic reviews (SysRev) are being published in health sciences and medicine; however, many are poorly conducted or reported (Glasziou et al., 2014). Strategies are needed to help reduce this avoidable waste in research (loannidis et al., 2014; Moher et al., 2016; Moher et al., 2017). Systematic reviews can help decision makers interpret the deluge of published biomedical literature. However, a SysRev or scoping review may be of limited use if the methods used to conduct them are flawed, or if reporting is incomplete. (Catalá et al., 2016).

At each stage during the systematic or scoping review cycle, different challenges can arise, especially for a novice researcher. Some of these challenges include poor or limited training in:

1. Research methods

Early career researchers (ECRs) often struggle with mapping their question to an appropriate review methodology, or they struggle with the components of the methodology, including the timelines, team members, resources, project design, and reporting.

2. Standards

Methodologies and reporting guidelines are frequently being updated, changed or developed to address different questions. Researchers, especially ECRs, struggle keeping abreast of all the new developments in their field.

3. Publication

Publishing presents a steep learning curve as guidelines vary from journal to journal. However, there are common overarching principles, which remain unknown to a vast majority of ECRs.

\section{Rationale}

All knowledge syntheses, once past the stage of question formulation, begin with the literature search. Librarians are in a strategic position to uncover issues regarding a researcher's level of preparedness in conducting these types of studies. From this vantage point, librarians can have a significant impact by teaching researchers about practices to properly report findings, as well as by raising awareness about which methodology might be more appropriate for their research question (Edmunds Otter, Wright, \& King, 2017; Heimlich, 2014; Meert, Torabi, \& Costella, John, 2016). Research waste is a growing concern (Simera et al., 2010), and librarians are part of the answer (Kirtley, 2016) in the role they play as advocates for research 
integrity and transparency. Librarians can also act as educators in showcasing tools to our clients that can facilitate the attainment of these principles.

This scoping review would be the first to cover this topic in a comprehensive, structured and methodologically rigorous way. Results would be of interest to librarians, researchers, educators and the wider research community in health sciences and medicine.

\section{Definitions}

\section{What are systematic and scoping reviews?}

A systematic review (SysRev) attempts to identify, appraise and synthesize all the empirical evidence that meets pre-specified eligibility criteria to answer a given research question. Researchers conducting systematic reviews use explicit methods aimed at minimizing bias, in order to produce more reliable findings that can be used to inform decision making. (Higgins \& Green, 2011)

A scoping review (ScopRev) or scoping study is a form of knowledge synthesis that addresses an exploratory research question aimed at mapping key concepts, types of evidence, and gaps in research related to a defined area or field by systematically searching, selecting, and synthesizing existing knowledge. (Colquhoun et al., 2014)

\section{Who are early career researchers (ECRs)?}

Early career researchers (ECRs) are "typically assistant professors at research universities and hospitals who have held their positions for 5 years or less" (http://www.acechr.ca/ about.html). For the purposes of this study, graduate students (including Masters and PhD candidates, as well as postdoctoral fellows) will be included as early career researchers, as they are learning about these research methods at this stage of their career.

\section{Which topics are considered health sciences and medicine?}

The following topics are considered health sciences and medicine: neuroscience, pharmacology, nutritional sciences, physiotherapy, kinesiology, public health, population health, radiation therapy, life sciences, biomedicine, dentistry, health management, epidemiology, virology, rehabilitation, speech language pathology, nursing, midwifery, occupational therapy, psychology, social work, psychotherapy, chiropractor, acupuncture, all medical specialties and all alternative forms of therapies.

\section{Objectives}

Primary objective: To systematically discover, collect and synthesize the challenges, opportunities and level of preparedness of ECRs when conducting scoping and systematic reviews in the health sciences. So far, the evidence consists of individual reports, case studies and anecdotal data.

Secondary objective: To raise awareness about the challenges faced by ECRs, and to work with other librarians and stakeholders on ways to address these challenges, leverage existing opportunities and develop new ones.

Tertiary objective: To enhance librarian support to ECRs through an improved understanding 
of the challenges that ECRs face at each step of the review process, and also by developing ways to overcome these challenges to facilitate how ECRs undertake systematic or scoping review processes. The results of this study would benefit the entire university community and affiliated institutions, and strengthen or develop relationships among faculties, administrators and staff to find creative solutions that benefits ECRs in the undertaking of scoping and systematic reviews.

\section{Methods}

This scoping review will follow the methodological framework described by Arksey and O'Malley (2005), along with refinements proposed to this framework by Colquhoun et al. (2014), as well as the PRISMA-ScR statement. This six-stage model involves: (1) identifying the research question (listed above); (2) identifying relevant studies (search methods used); (3) selecting studies; (4) charting the data; (5) collating, summarizing and reporting the results and (6) consulting with key stakeholders. The refinements to the original framework include establishing a clear research question, purpose and outcome of the scoping review; assembling a team with content and methodological expertise; searching the literature using an iterative process with inclusion and exclusion criteria; using at least two reviewers to independently review abstracts and full-text papers, with a consensus procedure in case of a disagreement; developing a data abstraction form where two researchers abstract the data independently; and performing an analysis which includes a descriptive numerical summary of papers as well as a qualitative thematic analysis. Finally, consultation with key stakeholders is a required step in a scoping review and is not optional.

\section{Eligibility criteria}

\section{Inclusion criteria}

We will include studies that examine the conduct of scoping or systematic reviews, and early career researchers in the health sciences. All publication types, with the exception of knowledge syntheses, will be considered provided the studies are based on empirical research.

\section{Exclusion criteria}

We will exclude editorials, commentaries, any comprehensive literature review as publication types, and studies that do not examine the systematic or scoping review cycle as their main outcome. We will also exclude any studies that refer only to early career researchers outside of the health sciences.

\section{Information sources and literature search}

\section{Search Strategy}

Comprehensive literature searches will be conducted in the following databases: Medline (via OVID), Embase (via Ovid), CINAHL (via EBSCOHost), PsycINFO (via Ovid), ERIC (via Ovid), Education Source (via EBSCOHost), and Library, Information and Technology Abstracts -LISTA (via EBSCOHost). The databases will be searched from their dates of inception to June 15th, 2018. Search strategies will be developed by an academic health sciences librarian (LS), and will be peer reviewed using the Peer Review of Electronic Search Strategies (PRESS) tool. We will crowdsource synonyms for the term "early career researcher" from social platforms including Facebook and Twitter, while concurrently searching the literature. We will adapt the "system- 
atic review" search filter from the Scottish Intercollegiate Guidelines Network (SIGN) when searching for SRs and scoping reviews as a topic.

Supplemental searching will include cited reference searching and citation analysis using Web of Science and Scopus. Additionally, we will search the grey literature and international policies using standardized scoping review methodologies.

\section{Study selection}

\section{Study selection process}

The team will perform a calibration exercise based on the eligibility criteria using a random sample of 6 titles and abstracts screened independently by each reviewer. Two calibration exercises (using 6 records each time) will be performed by the team with the goal of reaching $90 \%$ agreement after clarifications on eligibility criteria are discussed. Disagreements will be resolved by discussion amongst pairs of reviewers or with a third member, if required. The same process will be followed for full-text screening, with 6 random full-text articles used to achieve $90 \%$ agreement.

Once results from the calibration exercise are satisfactory, at least two reviewers will independently screen all titles and abstracts identified by the literature search. Full-text articles for potentially eligible studies will be obtained and screened independently by pairs of reviewers using the same eligibility criteria as with the title and abstract screening. Covidence, an online screening tool, will be used for the first and second levels of screening.

\section{Data items and data collection process}

Two reviewers will independently assess each eligible study using a structured data extraction form (Appendix 3). The reviewers will then compare their decisions to identify and discuss discrepancies, which will be resolved through consensus. If consensus cannot be reached between the two reviewers, a third member of the review team will be consulted to resolve the disagreement.

We will conduct a descriptive analysis on variables of interest from all included studies to provide information regarding participant, setting and exposure characteristics for all studies that met the eligibility criteria.

A data management plan will be developed using an online DMPTool. https://dmptool.org/

\section{Synthesis of included studies}

To synthesize our results, we will summarize all of the abstracted data. For example, we will provide overall summaries for each of the following: study characteristics, participant characteristics, and sample characteristics. We will observe the number of studies available for each of the outcomes examined, study designs by outcome, as well as the total number of participants included in the studies that report each outcome.

\section{Challenges}

Varying nomenclature and definition of SRs 
We acknowledge that there are varying definitions and naming conventions for these knowledge syntheses types, particularly for scoping reviews (Peterson, J. Et al, 2017; Colquhoun et al, 2014; O’Brien et al., 2016). Literature reviews, scoping studies, scoping analysis, to name a few, are terms frequently used interchangeably for systematic and scoping reviews, and pose a challenge in identifying studies of interest during the first level of screening. To mitigate this, we will include all studies that mention the varying terms during the first level of screening, unless they can be clearly excluded.

\title{
Limitations
}

\section{Language}

We will acknowledge the limitation of only including studies in English. Despite the limitations mentioned above, the proposed systematic review is of value both theoretically and practically.

\section{Discussion and Dissemination}

In addition to informing systematic and scoping review methodology best practices within the academic research community, this review will generate results that will be relevant to the broader health sciences and medical community. The results will help map the literature and identify gaps where primary evidence is lacking. We anticipate our scoping review will identify gaps in training, mentorship, and other opportunities and challenges. All data in this project will be gathered through searches of literature databases and policies available online. No personal (health) information will be collected in the context of this project. Therefore, approval from a research ethics committee is not required.

This review will provide an overview of which interventions and policies are effective, and will assist educators, researchers, and faculty, by helping them make informed decisions regarding implementation within their organizations. Findings will be shared with educators, researchers and ECRs at our stakeholder meetings, and will be disseminated to other local, national and international stakeholders at relevant meetings and conferences, and publication through open access platforms.

\section{Report}

The reporting of this protocol is based on guidance from the Preferred Reporting Items for Systematic reviews and Meta-analyses Protocols (PRISMA-P), the checklist can be found in Appendix 4. All documents (cheat sheets, calibration exercises, author information, PRESS, and more) can be found in https://osf.io/zdm3c/

\author{
Abbreviations \\ ECRs: Early career researchers \\ PRESS: Peer Review of Electronic Search Strategies \\ PRISMA-P: Preferred reporting items for systematic reviews and meta-analyses protocols \\ PRISMA-ScR: Preferred reporting items for scoping reviews \\ SysRev: Systematic Review \\ ScopRev: Scoping Review
}




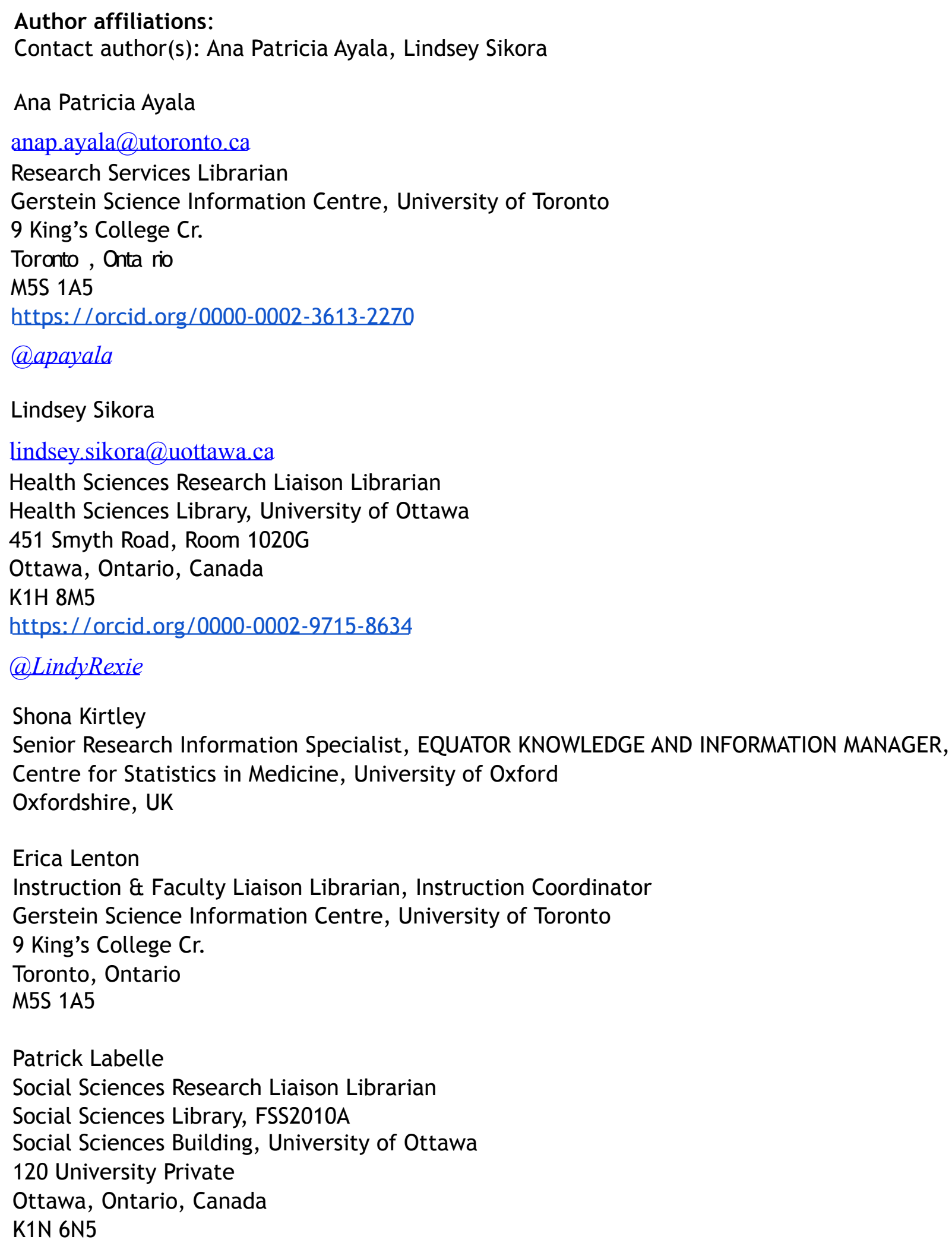

Authors' contributions: All authors participated in the design and development of the protocol. APA, LS, SK, EL, PL drafted the manuscript, and all authors read and approved the final manuscript. 
Competing interests: The authors report no competing interests.

Acknowledgements: Our team is grateful to Kaitlin Fuller, who conducted a PRESS review on our search strategies.

Funding: None

Ethics approval: Not required

Provenance and peer review: Not commissioned

\section{References}

Arksey, H., \& O'Malley, L. (2005). Scoping studies: Towards a methodological framework. International Journal of Social Research Methodology, 8(1), 19-32. doi: $10.1080 / 1364557032000119616$

CASRAI. CASRAI CRediT; Retrieved February 27, 2018, from http://docs.casrai.org/CRediT

Catalá-López, F., Moher, D., \& Tabars-Seisdedos, R. (2016). Improving transparency of scientific reporting to increase value and reduce waste in mental health research. Revista De Psiquiatria Y Salud Mental, 9(1), 1-3. doi:10.1016/j.rpsm.2016.01.002

Colquhoun, H. L., Levac, D., O'Brien, K. K., Straus, S., Tricco, A. C., Perrier, L., et al. (2014). Scoping reviews: Time for clarity in definition, methods, and reporting. Journal of Clinical Epidemiology, 67(12), 1291-1294. doi:10.1016/j.jclinepi.2014.03.013

Edmunds Otter, M. L., Wright, J. M., \& King, N. V. (2017). Developing the librarians' role in supporting grant applications and reducing waste in research: Outcomes from a literature review and survey in the NIHR research design service. New Review of Academic Librarianship, 23(2-3), 258-274. doi:10.1080/13614533.2017.1330219

Glasziou, P., Altman, D. G., Bossuyt, P., Boutron, I., Clarke, M., Julious, S., et al. (2014). Reducing waste from incomplete or unusable reports of biomedical research. The Lancet, 383(9913), 267-276. doi:10.1016/S0140-6736(13)62228-X

Heimlich, S. L. (2014). New and emerging roles for medical librarians. Journal of Hospital Librarianship, 14(1), 24-32. doi:10.1080/15323269.2014.859995

Higgins, J., \& Green, S. (2011). Cochrane handbook for systematic reviews of interventions version 5.1.0\&nbsp; 2011.\&nbsp;. Available from http://handbook.cochrane.org.myaccess.library.utoronto.ca: The Cochrane Collaboration. 
loannidis, J. P. A., Greenland, S., Hlatky, M. A., Khoury, M. J., Macleod, M. R., Moher, D., et al. (2014). Increasing value and reducing waste in research design, conduct, and analysis. The Lancet, 383(9912), 166-175. doi:10.1016/S0140-6736(13)62227-8

Kirtley, S. (2016). Increasing value and reducing waste in biomedical research: Librarians are listening and are part of the answer doi://doi-org.myaccess.library.utoronto.ca/ 10.1016/S0140-6736(16)30241-0"

Meert, D., MLIS, Torabi, N., MLIS, \& Costella, John, DDS,MSc, MLIS. (2016). Impact of librarians on reporting of the literature searching component of pediatric systematic reviews *. Journal of the Medical Library Association, 104(4), 267-277. doi://dx.doi.org.myaccess.library.utoronto.ca/10.3163/1536-5050.104.4.004

Moher, D., Glasziou, P., Chalmers, I., Nasser, M., Bossuyt, P. M. M., Korevaar, D. A., et al. (2016). Increasing value and reducing waste in biomedical research: Who's listening? The Lancet, 387(10027), 1573-1586. doi:10.1016/S0140-6736(15)00307-4

Moher, D., Shamseer, L., Cobey, K., Lalu, M. M., Galipeau, J., Avey, M. T., et al. (2017). Stop this waste of people, animals and money. Nature, 549(7670), 23-25. doi: $10.1038 / 549023 a$

O'Brien, K. K., Colquhoun, H., Levac, D., Baxter, L., Tricco, A. C., Straus, S., et al. (2016). Advancing scoping study methodology: A web-based survey and consultation of perceptions on terminology, definition and methodological steps. BMC Health Services Research, 16(1) doi:10.1186/s12913-016-1579-z

Open science framework. (2015). Center for Open Science (COS).

Page, M. J., Shamseer, L., Altman, D. G., Tetzlaff, J., Sampson, M., Tricco, A. C., et al. (2016). Epidemiology and reporting characteristics of systematic reviews of biomedical research: A cross-sectional study. PLoS Medicine, 13(5) doi:10.1371/journal.pmed. 1002028

Peterson, J., Pearce, P. F., Ferguson, L. A., \& Langford, C. A. (2017). Understanding scoping reviews. Journal of the American Association of Nurse Practitioners, 29(1), 12-16. doi: $10.1002 / 2327-6924.12380$

Pfeffermann, D., \& Rao, C. R. (2010). Sample surveys: Design, methods and applications. Amsterdam: Elsevier.

Pham MT, Rajić A, Greig JD, Sargeant JM, Papadopoulos A, McEwen SA. A scoping review of scoping reviews: advancing the approach and enhancing the consistency. Research Synthesis Methods. 2014;5(4):371-385. doi:10.1002/jrsm.1123.

Simera, I., Moher, D., Hirst, A., Hoey, J., Schulz, K. F., \& Altman, D. G. (2010). Transparent and accurate reporting increases reliability, utility, and impact of your research: Reporting guidelines and the EQUATOR network. BMC Medicine, 8 doi:10.1186/1741-7015-8-24 
Prepared by: Patricia Ayala and Lindsey Sikora Date: October 22, 2018

Version: 6

Last updated: December 10, 2019 


\begin{tabular}{l|l}
\hline Literature Database & Discipline or Area of Study \\
\hline ERIC (ProQuest) & $\begin{array}{l}\text { Education; Kinesiology; Physical Education; } \\
\text { Speech Language Pathology }\end{array}$ \\
\hline CINAHL & Nursing and allied health \\
\hline
\end{tabular}

Medline (OVID)

PsycINFO (OVID)

EMBASE (OVID)
Bioethics; Biophysics; Dentistry; Forensic

Science; Genetics; Gerontology and

Geriatrics; Health Administration;

Kinesiology; Medicine; Neuroscience;

Nursing; Nutrition; Occupational Therapy;

Pharmacology; Pharmacy; Physical

Education; Physical Therapy; Physiology;

Psychiatry; Public Health; Rehabilitation

Sciences; Speech Language Pathology;

Toxicology; Veterinary Sciences

Criminology; Forensic Science;

Gerontology and Geriatrics; Kinesiology;

Medicine; Neuroscience; Physical

Education; Psychiatry; Psychology;

Speech Language Pathology; Substance

Abuse

Pharmacology and toxicology, General

clinical medicine, Genetics, biochemistry

\& molecular biology, Neurology \&

behavioral medicine, Microbiology \&

infectious disease, Cardiology \&

hematology, Psychiatry \& mental health

Oncology, Healthcare policy \&

management

Allergy \& immunology, Pediatrics, Endocrinology \& metabolism, Obstetrics \& gynecology, Biomedical engineering \& medical devices, Anesthesiology \& intensive care, Gastroenterology,

Respiratory medicine

Nephrology \& urology, Dermatology,

Geriatrics \& gerontology 


\begin{tabular}{l|l}
\hline Education Source & Education \\
\hline $\begin{array}{l}\text { Library, Information and Technology } \\
\text { Abstracts (LISTA) }\end{array}$ & Library and Information Science \\
\hline
\end{tabular}

\section{Appendix 2: MEDLINE Search Strategy}

1. Meta-Analysis as Topic/

2. meta analy\$.tw,kf.

3. metaanaly\$.tw,kf.

4. Meta-Analysis/

5. ((scoping or systematic) adj3 (review\$1 or overview\$1 or study or studies or analys*)).tw,kf.

6. exp Review Literature as Topic/

7. or/1-6

8. (early adj2 career*).tw,kf.

9. (entry adj2 level*).tw,kf.

10. pre?intern*.tw,kf.

11. (new adj3 career*).tw,kf.

12. ((emerging* ${ }^{*}$ or emergent ${ }^{*}$ or early stage or junior* or early career* or new or recent* or young or novice* or start* or beginner* or newcomer* or newly qualified) adj3 (investigator* or trainee* or researcher* or intern* or graduat* or prof* or facult ${ }^{*}$ or lecturer* or fellow* or scientist* or academic*)).tw,kf.

13. ECR*.tw,kf.

14. (research adj3 (assistant* or associate* or coordinator*)).tw,kf.

15. (post adj3 universit*).tw,kf.

16. pre?tenur*.tw,kf.

17. newbie ${ }^{*} . t w, k f$.

18. exp Education, Graduate/

19. $\left(\right.$ grad $^{*} \operatorname{adj} 3$ (student* or education*)).tw,kf.

20. Foreign Medical Graduates/

21. post?doc*.tw,kf.

22. exp Internship, Nonmedical/

23. post?universit*. ${ }^{*} \mathrm{w}, \mathrm{kf}$.

24. or/8-23

25. 7 and 24 


\section{Appendix 3: Components of Data Abstraction Form}

1. Study characteristics (reference ID, first author; year of publication; article title, journal name, study design(s), methodology, study period)

2. Setting \& objectives (study purpose/objective, implementation, physical or geographical location, setting (organisation type), associated discipline(s))

3. Population or sample characteristics (participant characteristics: age, role, gender, level of education, sample size, sample characteristics)

4. Data collection tools or outcome measures (survey, interviews, scales, instruments)

5. Outcomes (outcomes, results)

\section{Appendix 4: PRISMA-P Checklist}

This checklist has been adapted for use with scoping review protocol submissions to OSF PrePrints from Table 3 in Moher $D$ et al: Preferred reporting items for systematic review and meta-analysis protocols (PRISMA-P) 2015 statement. Systematic Reviews 2015 4:1

An Editorial from the Editors-in-Chief of Systematic Reviews details why this checklist was adapted - Moher D, Stewart L \& Shekelle P: Implementing PRISMA-P: recommendations for prospective authors. Systematic Reviews 2016 5:15

\begin{tabular}{|c|c|c|c|c|c|}
\hline \multirow[t]{2}{*}{ Section/topic } & \multirow[t]{2}{*}{$\#$} & \multirow[t]{2}{*}{ Checklist item } & \multicolumn{2}{|c|}{$\begin{array}{l}\text { Information } \\
\text { reported }\end{array}$} & \multirow{2}{*}{$\begin{array}{l}\text { Line } \\
\text { number(s) }\end{array}$} \\
\hline & & & Yes & No & \\
\hline \multicolumn{6}{|c|}{ ADMINISTRATIVE INFORMATION } \\
\hline \multicolumn{6}{|l|}{ Title } \\
\hline Identification & $1 \mathrm{a}$ & $\begin{array}{l}\text { Identify the report as a protocol of a } \\
\text { systematic (in this case scoping) review }\end{array}$ & $x$ & & $3-4$ \\
\hline Update & $1 b$ & $\begin{array}{l}\text { If the protocol is for an update of a previous } \\
\text { systematic review, identify as such }\end{array}$ & & $\mathbf{x}$ & N/A \\
\hline Registration & 2 & $\begin{array}{l}\text { If registered, provide the name of the registry } \\
\text { (e.g., PROSPERO) and registration number } \\
\text { in the Abstract }\end{array}$ & $x$ & & \\
\hline \multicolumn{6}{|l|}{ Authors } \\
\hline Contact & $3 a$ & $\begin{array}{l}\text { Provide name, institutional affiliation, and e- } \\
\text { mail address of all protocol authors; provide } \\
\text { physical mailing address of corresponding } \\
\text { author }\end{array}$ & $x$ & & 6 \\
\hline Contributions & $3 b$ & $\begin{array}{l}\text { Describe contributions of protocol authors } \\
\text { and identify the guarantor of the review }\end{array}$ & $x$ & & 6 \\
\hline
\end{tabular}




\begin{tabular}{|c|c|c|c|c|c|}
\hline \multirow{2}{*}{ Section/topic } & \multirow[t]{2}{*}{$\#$} & \multirow{2}{*}{ Checklist item } & \multicolumn{2}{|c|}{$\begin{array}{l}\text { Information } \\
\text { reported }\end{array}$} & \multirow{2}{*}{$\begin{array}{l}\text { Line } \\
\text { number(s) }\end{array}$} \\
\hline & & & Yes & No & \\
\hline Amendments & 4 & $\begin{array}{l}\text { If the protocol represents an amendment of a } \\
\text { previously completed or published protocol, } \\
\text { identify as such and list changes; otherwise, } \\
\text { state plan for documenting important } \\
\text { protocol amendments }\end{array}$ & & $x$ & N/A \\
\hline \multicolumn{6}{|l|}{ Support } \\
\hline Sources & $5 a$ & $\begin{array}{l}\text { Indicate sources of financial or other support } \\
\text { for the review }\end{array}$ & & $x$ & $N / A$ \\
\hline Sponsor & $5 b$ & $\begin{array}{l}\text { Provide name for the review funder and/or } \\
\text { sponsor }\end{array}$ & & $x$ & N/A \\
\hline $\begin{array}{c}\text { Role of } \\
\text { sponsor/funder }\end{array}$ & $5 c$ & $\begin{array}{l}\text { Describe roles of funder(s), sponsor(s), and/ } \\
\text { or institution(s), if any, in developing the } \\
\text { protocol }\end{array}$ & & $x$ & N/A \\
\hline \multicolumn{6}{|l|}{ INTRODUCTION } \\
\hline Rationale & 6 & $\begin{array}{l}\text { Describe the rationale for the review in the } \\
\text { context of what is already known }\end{array}$ & $x$ & & $1-2$ \\
\hline Objectives & 7 & $\begin{array}{l}\text { Provide an explicit statement of the } \\
\text { question(s) the review will address with } \\
\text { reference to participants, interventions, } \\
\text { comparators, and outcomes (PICO) }\end{array}$ & $x$ & & 2 \\
\hline \multicolumn{6}{|l|}{ METHODS } \\
\hline Eligibility criteria & 8 & $\begin{array}{l}\text { Specify the study characteristics (e.g., PICO, } \\
\text { study design, setting, time frame) and report } \\
\text { characteristics (e.g., years considered, } \\
\text { language, publication status) to be used as } \\
\text { criteria for eligibility for the review }\end{array}$ & $x$ & & 3 \\
\hline $\begin{array}{l}\text { Information } \\
\text { sources }\end{array}$ & 9 & $\begin{array}{l}\text { Describe all intended information sources } \\
\text { (e.g., electronic databases, contact with } \\
\text { study authors, trial registers, or other grey } \\
\text { literature sources) with planned dates of } \\
\text { coverage }\end{array}$ & $x$ & & $3-4$ \\
\hline Search strategy & 10 & $\begin{array}{l}\text { Present draft of search strategy to be used } \\
\text { for at least one electronic database, } \\
\text { including planned limits, such that it could be } \\
\text { repeated }\end{array}$ & $x$ & & 11 \\
\hline \multicolumn{6}{|l|}{ STUDY RECORDS } \\
\hline $\begin{array}{c}\text { Data } \\
\text { management }\end{array}$ & $\begin{array}{l}11 \\
a\end{array}$ & $\begin{array}{l}\text { Describe the mechanism(s) that will be used } \\
\text { to manage records and data throughout the } \\
\text { review }\end{array}$ & $x$ & & 4 \\
\hline
\end{tabular}




\begin{tabular}{|c|c|c|c|c|c|}
\hline \multirow{2}{*}{ Section/topic } & \multirow[t]{2}{*}{$\#$} & \multirow{2}{*}{ Checklist item } & \multicolumn{2}{|c|}{$\begin{array}{l}\text { Information } \\
\text { reported }\end{array}$} & \multirow{2}{*}{$\begin{array}{l}\text { Line } \\
\text { number(s) }\end{array}$} \\
\hline & & & Yes & No & \\
\hline $\begin{array}{l}\text { Selection } \\
\text { process }\end{array}$ & $\begin{array}{l}11 \\
b\end{array}$ & $\begin{array}{l}\text { State the process that will be used for } \\
\text { selecting studies (e.g., two independent } \\
\text { reviewers) through each phase of the review } \\
\text { (i.e., screening, eligibility, and inclusion in } \\
\text { meta-analysis) }\end{array}$ & $x$ & & 4 \\
\hline $\begin{array}{l}\text { Data } \\
\text { collection process }\end{array}$ & $\begin{array}{l}11 \\
\mathrm{C}\end{array}$ & $\begin{array}{l}\text { Describe planned method of extracting data } \\
\text { from reports (e.g., piloting forms, done } \\
\text { independently, in duplicate), any processes } \\
\text { for obtaining and confirming data from } \\
\text { investigators }\end{array}$ & $x$ & & 4 \\
\hline Data items & 12 & $\begin{array}{l}\text { List and define all variables for which data } \\
\text { will be sought (e.g., PICO items, funding } \\
\text { sources), any pre-planned data assumptions } \\
\text { and simplifications }\end{array}$ & $x$ & & $11-12$ \\
\hline $\begin{array}{l}\text { Outcomes and } \\
\text { prioritization }\end{array}$ & 13 & $\begin{array}{l}\text { List and define all outcomes for which data } \\
\text { will be sought, including prioritization of main } \\
\text { and additional outcomes, with rationale }\end{array}$ & $x$ & & $11-12$ \\
\hline $\begin{array}{l}\text { Risk of bias in } \\
\text { individual } \\
\text { studies }\end{array}$ & 14 & $\begin{array}{l}\text { Describe anticipated methods for assessing } \\
\text { risk of bias of individual studies, including } \\
\text { whether this will be done at the outcome or } \\
\text { study level, or both; state how this } \\
\text { information will be used in data synthesis }\end{array}$ & & $x$ & N/A \\
\hline \multicolumn{6}{|l|}{ DATA } \\
\hline \multirow{4}{*}{ Synthesis } & $\begin{array}{l}15 \\
a\end{array}$ & $\begin{array}{l}\text { Describe criteria under which study data will } \\
\text { be quantitatively synthesized }\end{array}$ & & $X$ & N/A \\
\hline & $\begin{array}{l}15 \\
b\end{array}$ & $\begin{array}{l}\text { If data are appropriate for quantitative } \\
\text { synthesis, describe planned summary } \\
\text { measures, methods of handling data, and } \\
\text { methods of combining data from studies, } \\
\text { including any planned exploration of } \\
\text { consistency (e.g., I } 2 \text {, Kendall's tau) }\end{array}$ & & $x$ & N/A \\
\hline & $\begin{array}{l}15 \\
\mathrm{c}\end{array}$ & $\begin{array}{l}\text { Describe any proposed additional analyses } \\
\text { (e.g., sensitivity or subgroup analyses, meta- } \\
\text { regression) }\end{array}$ & & $X$ & N/A \\
\hline & 15 & $\begin{array}{l}\text { If quantitative synthesis is not appropriate, } \\
\text { describe the type of summary planned }\end{array}$ & $x$ & & $4-5$ \\
\hline Meta-bias(es) & 16 & $\begin{array}{l}\text { Specify any planned assessment of meta- } \\
\text { bias(es) (e.g., publication bias across } \\
\text { studies, selective reporting within studies) }\end{array}$ & & $x$ & $N / A$ \\
\hline $\begin{array}{l}\text { Confidence in } \\
\text { cumulative } \\
\text { evidence }\end{array}$ & 17 & $\begin{array}{l}\text { Describe how the strength of the body of } \\
\text { evidence will be assessed (e.g., GRADE) }\end{array}$ & & $x$ & $N / A$ \\
\hline
\end{tabular}

